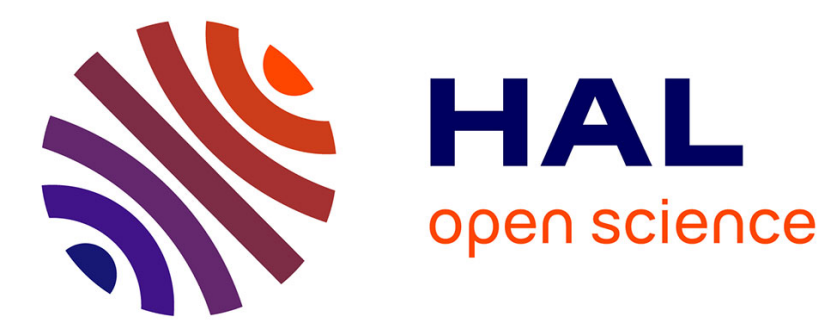

\title{
Asymptotic analysis of beamspace-MUSIC in the context of large arrays
}

Pascal Vallet, Xavier Mestre, Philippe Loubaton, Romain Couillet

\section{To cite this version:}

Pascal Vallet, Xavier Mestre, Philippe Loubaton, Romain Couillet. Asymptotic analysis of beamspaceMUSIC in the context of large arrays. 2014 IEEE 8th Sensor Array and Multichannel Signal Processing Workshop (SAM) , Jun 2014, La Coruna, Spain. pp.469-472, 10.1109/SAM.2014.6882444 . hal01618550

\section{HAL Id: hal-01618550 https://hal.science/hal-01618550}

Submitted on 18 Oct 2017

HAL is a multi-disciplinary open access archive for the deposit and dissemination of scientific research documents, whether they are published or not. The documents may come from teaching and research institutions in France or abroad, or from public or private research centers.
L'archive ouverte pluridisciplinaire HAL, est destinée au dépôt et à la diffusion de documents scientifiques de niveau recherche, publiés ou non, émanant des établissements d'enseignement et de recherche français ou étrangers, des laboratoires publics ou privés. 


\title{
Asymptotic analysis of Beamspace-MUSIC in the context of large arrays
}

\author{
Pascal Vallet ${ }^{*}$, Xavier Mestre ${ }^{\dagger}$, Philippe Loubaton ${ }^{\ddagger}$, Romain $_{\text {Couillet }}{ }^{\S}$ \\ * Laboratoire IMS (CNRS, Univ. Bordeaux I, IPB), 351 Cours de la Libération, 33400 Talence, France \\ $\dagger$ Centre Tecnològic de Telecomunicacions de Catalunya (CTTC), Av. Carl Friedrich Gauss 08860 Castelldefels, Spain \\ $\ddagger$ Laboratoire IGM (CNRS, Univ. Paris-Est/MLV), 5 Boulevard Descartes, 77454 Marne-la-Vallée, France \\ $\S$ Supélec, 3 rue Joliot Curie, 91192 Gif-sur-Yvette, France \\ pascal.vallet@ipb.fr, loubaton@univ-mlv.fr, xavier.mestre@cttc.cat, romain.couillet@supelec.fr
}

\begin{abstract}
It is well-known that the MUSIC method for DoA estimation degrades when the number of samples $N$ and the array dimension $M$ are large and of the same order of magnitude. In this context, several improvements have been proposed, among which the G-MUSIC method, which was shown to be consistent in the asymptotic regime where $M, N$ converge to infinity at the same rate, and under an additional separation condition between noise and signal subspaces of the SCM. Nevertheless, this subspace separation condition is only fulfilled for sufficiently high SNR. Dimension reduction techniques are a classical way to partially circumvent this condition. In this paper, we provide an asymptotic analysis in terms of consistency and MSE in the aforementioned regime, of the Beamspace MUSIC, which is one popular technique to reduce the dimension of the observations.
\end{abstract}

\section{INTRODUCTION}

The problem of estimating the Direction of Arrival (DoA) of $K$ source signals from a set of $N$ noisy observations collected by an array of $M$ sensors has been extensively studied in the past. Among the multiple algorithms that have been proposed, subspace methods, in particular MUSIC, are widely considered because they offer a good performance for a reduced computational cost, compared the maximum likelihood based method. We consider here the situation where $K$ narrowband and far-field source signals are impinging on an array of $M$ sensors $(K<M)$, with DoA $\theta_{1}, \ldots, \theta_{K}$. At discrete time $n$, the received signal $\mathbf{y}_{n} \in \mathbb{C}^{M}$ is usually modeled as

$$
\mathbf{y}_{n}=\mathbf{A} \mathbf{s}_{n}+\mathbf{v}_{n}
$$

where $\mathbf{A}=\left[\mathbf{a}\left(\theta_{1}\right), \ldots, \mathbf{a}\left(\theta_{K}\right)\right]$ is the matrix containing the steering vectors $\mathbf{a}\left(\theta_{1}\right), \ldots, \mathbf{a}\left(\theta_{K}\right)$, with $\mathbf{a}(\theta)=$ $M^{-1 / 2}\left[1, \ldots, \mathrm{e}^{\mathrm{i}(M-1) \theta}\right]^{T}$, where $\mathbf{s}_{n} \in \mathbb{C}^{K}$ contains the $K$ source signals received at time $n$, and where $\mathbf{v}_{n}$ is a complex, additive, spatially and temporally white Gaussian noise, with $\mathbb{E}\left[\mathbf{v}_{n} \mathbf{v}_{n}^{*}\right]=\sigma^{2} \mathbf{I}$. When $N$ samples are collected in the matrix $\mathbf{Y}_{N}=\left[\mathbf{y}_{1}, \ldots, \mathbf{y}_{N}\right]$, the previous model then writes

$$
\mathbf{Y}_{N}=\mathbf{A} \mathbf{S}_{N}+\mathbf{V}_{N}
$$

where $\mathbf{S}_{N}=\left[\mathbf{s}_{1}, \ldots, \mathbf{s}_{N}\right]$ et $\mathbf{V}_{N}=\left[\mathbf{v}_{1}, \ldots, \mathbf{v}_{N}\right]$. In the remainder, we consider the so-called conditional assumption, where the source signals are assumed (unknown) deterministic.

This work was partially supported by GDR ISIS and GRETSI ("Projet Jeunes Chercheurs").
When $\operatorname{rank}\left(\mathbf{S}_{N}\right)=K$, the DoA $\theta_{1}, \ldots, \theta_{K}$ are zeros of the pseudo-spectrum function

$$
\eta_{N}(\theta)=\mathbf{a}(\theta)^{*} \boldsymbol{\Pi}_{N} \mathbf{a}(\theta),
$$

where $\boldsymbol{\Pi}_{N}$ is the orthogonal projection matrix onto the noise subspace (i.e. the kernel of $\mathbf{A} \mathbf{S}_{N} \mathbf{S}_{N}^{*} \mathbf{A}^{*}$ ). Since $\boldsymbol{\Pi}_{N}$ is not available in practice, its standard estimate $\hat{\boldsymbol{\Pi}}_{N}$ is obtained by computing the orthogonal projection matrix onto the eigenspace associated with the $M-K$ smallest eigenvalues of the sample covariance matrix (SCM)

$$
\frac{\mathbf{Y}_{N} \mathbf{Y}_{N}^{*}}{N}=\frac{1}{N} \sum_{n=1}^{N} \mathbf{y}_{n} \mathbf{y}_{n}^{*} .
$$

The MUSIC algorithm then consists in estimating $\theta_{1}, \ldots, \theta_{K}$ as the $K$ most significant minima of

$$
\hat{\eta}_{N}(\theta)=\mathbf{a}(\theta)^{*} \hat{\mathbf{\Pi}}_{N} \mathbf{a}(\theta) .
$$

The use of $\hat{\boldsymbol{\Pi}}_{N}$ is motivated by the fact that (law of large number)

$$
\left\|\frac{\mathbf{Y}_{N} \mathbf{Y}_{N}^{*}}{N}-\mathbf{R}_{N}\right\| \rightarrow 0,
$$

almost surely (a.s.), as $M$ is fixed and $N \rightarrow \infty$ (\|.\| stands for the spectral norm), where $\mathbf{R}_{N}=\mathbf{A} \frac{\mathbf{S}_{N} \mathbf{S}_{N}^{*}}{N} \mathbf{A}^{*}+\sigma^{2} \mathbf{I}$ is the covariance matrix of the observations. The convergence (5) implies $\left\|\hat{\boldsymbol{\Pi}}_{N}-\boldsymbol{\Pi}_{N}\right\| \rightarrow 0$ and

$$
\sup _{\theta \in[-\pi, \pi]}\left|\hat{\eta}_{N}(\theta)-\eta_{N}(\theta)\right| \underset{N \rightarrow \infty}{\stackrel{a . s .}{\longrightarrow}} 0,
$$

and therefore the consistency of the MUSIC DoA estimates. In practice, the use of the empirical estimate $\hat{\boldsymbol{\Pi}}_{N}$ makes sense whenever $N>>M$, and MUSIC performs relatively well in this context.

When the number of sensors $M$ is large and the signals are short-time stationary, the number of samples $N$ can be constrained to be of the same order of magnitude than $M$. In this regime, it is well-known that the performance of the MUSIC method severely degrades, essentially because the SCM is no more a good estimator of the true covariance $\mathbf{R}_{N}$. To model this new scenario, Mestre [1] consider a non-standard asymptotic regime in which $M$ and $N$ both 
converge to infinity at the same rate, that is $M, N \rightarrow \infty$ while $\frac{M}{N} \rightarrow c>0$. In this new asymptotic regime, (5) does not hold anymore, and consequently (4) does not estimate consistently (2). In particular, the eigenvalues of $\frac{\mathbf{Y}_{N} \mathbf{Y}_{N}^{*}}{N}$, instead of converging to the eigenvalues of $\mathbf{R}_{N}$, spread in several groups [2], resulting in a poor separation between noise and signal subspaces. Using results from random matrix theory, a "subspace separation condition" was derived in [2] (see also [1]), under which the $K$ largest sample eigenvalues split from the smallest $M-K$, resulting in a clear separation between noise and signal subspaces. Moreover, this condition was shown to hold for a SNR above a certain threshold. An extension of MUSIC (termed as "G-MUSIC") was then derived and shown to outperform the traditional MUSIC method for realistic values of $M, N$.

Nevertheless, the above subspace separation condition, which is inherent to the doubly asymptotic regime considered here, may be restrictive since it holds only at high SNR. One way to circumvent partially this drawback is to use dimensionality reduction techniques, which consist in virtually reducing the dimension of the observations $M$ by pre-processing the data $\mathbf{Y}_{N}$ before using subspace methods, in order to get back to a situation where the dimension of the observations is small compared to the number of samples $N$. Such techniques include in particular beamforming (see [3] and the references therein), spatial smoothing (see the recent reference [4]), or compressive sensing (see [5]).

In this paper, we propose a performance analysis of Beamspace-MUSIC, in the doubly asymptotic regime described above, when using a Discrete Fourier Transform (DFT) beamformer focused on a particular angular sector where the sources are suspected to lie. In particular, we prove the consistency and the asymptotic Gaussianity of the underlying DoA estimates, and provide an asymptotic analysis of the MSE. The paper is organized as follows. In section II, we provide some useful asymptotic results concerning the behaviour of the eigenvalues and eigenvectors of the SCM. In section III, we make use of the results of section II to study the performance of Beamspace MUSIC in terms of consistency and asymptotic MSE. Some numerical examples illustrate the previous results.

\section{ASYMPOTIC BEHAVIOUR OF THE S.C.M}

From now on, we assume the asymptotic regime where $M=M(N)$ is a function of $N$ such that $\frac{M}{N} \rightarrow c \in(0,1)$ as $N \rightarrow \infty$, and $K$ independent of $N$.

We recall below useful asymptotic results from random matrix theory, which will be useful for the subsequent analysis of Beamspace-MUSIC. For this, we consider a more general random matrix model

$$
\boldsymbol{\Sigma}_{N}=\mathbf{X}_{N}+\mathbf{W}_{N},
$$

where $\mathbf{X}_{N}$ is a rank $K$ deterministic matrix such that $\lim \sup _{N}\left\|\mathbf{X}_{N}\right\|<\infty$ and where $\mathbf{W}_{N}$ is a random matrix having i.i.d. zero-mean complex Gaussian entries with variance $\frac{\sigma^{2}}{N}$. Of course, model (6) encompass (1) by setting $\boldsymbol{\Sigma}_{N}=$ $N^{-1 / 2} \mathbf{Y}_{N}, \mathbf{X}_{N}=N^{-1 / 2} \mathbf{A} \mathbf{S}_{N}$ and $\mathbf{W}_{N}=N^{-1 / 2} \mathbf{V}_{N}$.
For the remainder, we denote by $\lambda_{1, N} \geq \ldots \geq \lambda_{K, N}$ the non-zero eigenvalues of $\mathbf{X}_{N} \mathbf{X}_{N}^{*}$ and by $\mathbf{u}_{1, N}, \ldots, \mathbf{u}_{K, N}$ the associated unit-norm eigenvectors. In the same way, we denote by $\hat{\lambda}_{1, N} \geq \ldots \geq \hat{\lambda}_{M, N}$ and $\hat{\mathbf{u}}_{1, N}, \ldots, \hat{\mathbf{u}}_{M, N}$ the eigenvalues and associated eigenvectors of $\boldsymbol{\Sigma}_{N} \boldsymbol{\Sigma}_{N}^{*}$.

Due to the non-standard asymptotic regime considered here, the following subspace separation condition [2] is needed to ensure an asymptotic separation between the signal and noise subspaces of the s.c.m. $\boldsymbol{\Sigma}_{N} \boldsymbol{\Sigma}_{N}^{*}$.

Assumption 1. For $k=1, \ldots, K, \lambda_{k, N} \rightarrow \lambda_{k}$ as $N \rightarrow \infty$, where $\lambda_{1} \geq \ldots \geq \lambda_{K}>\sigma^{2} \sqrt{c}$.

Indeed, under Assumption 1, we have for $k=1, \ldots, K$

$$
\hat{\lambda}_{k, N} \underset{N \rightarrow \infty}{\stackrel{a . s .}{\longrightarrow}} \frac{\left(\lambda_{k}+\sigma^{2} c\right)\left(\lambda_{k}+\sigma^{2}\right)}{\lambda_{k}}>\sigma^{2}(1+\sqrt{c})^{2},
$$

while $\hat{\lambda}_{K+1, N} \rightarrow \sigma^{2}(1+\sqrt{c})^{2}$ a.s. Thus, under the separation condition, the $K$ largest eigenvalues of $\boldsymbol{\Sigma}_{N} \boldsymbol{\Sigma}_{N}^{*}$ split asymptotically from the $M-K$ smallest, which implies the separation of the signal and noise subspaces. Moreover, under the subspace separation condition, we can characterize the asymptotic behaviour of any bilinear form of $\hat{\boldsymbol{\Pi}}_{N}$. For any sequences of unitary vectors $\left(\mathbf{d}_{1, N}\right),\left(\mathbf{d}_{2, N}\right)$, we then define

$$
\hat{\eta}_{N}=\mathbf{d}_{1, N}^{*}\left(\sum_{k=K+1}^{M} \hat{\mathbf{u}}_{k, N} \hat{\mathbf{u}}_{k, N}^{*}\right) \mathbf{d}_{2, N},
$$

and the following result holds.

Theorem 1. [6] Under Assumption 1, $\hat{\eta}_{N}=\eta_{N}+o(1)$ a.s., where

$$
\eta_{N}=\mathbf{d}_{1, N}^{*}\left(\mathbf{I}-\sum_{k=1}^{K} \gamma_{k, N} \mathbf{u}_{k, N} \mathbf{u}_{k, N}^{*}\right) \mathbf{d}_{2, N}
$$

with $\gamma_{k, N}=\frac{\lambda_{k, N}^{2}-\sigma^{4} c_{N}}{\lambda_{k, N}\left(\lambda_{k, N}+\sigma^{2} c_{N}\right)}$.

We now give a Central Limit Theorem (CLT) for $\hat{\eta}_{N}$ which can be obtained using the same steps as in [7]. Define ${ }^{1}$

$\vartheta_{N}(k, \ell)=-\frac{\lambda_{k, N} \lambda_{\ell, N}}{2} I_{1, N}(k, \ell)^{2}+$
$\frac{\sigma^{4}+\sigma^{2}\left(\lambda_{k, N}+\lambda_{\ell, N}\right)+\lambda_{k, N} \lambda_{\ell, N}}{2} \sum_{n \geq 0}\left(\sigma^{4} c_{N}\right)^{n} I_{n+1, N}(k, \ell)^{2}$,

where $I_{n, N}(k, \ell)$ is defined by the contour integral

$$
I_{n, N}(k, \ell)=\frac{1}{2 \pi \mathrm{i}} \oint_{\mathcal{C}} \frac{\left(w^{2}-\sigma^{4} c_{N}\right)\left(w+\sigma^{2} c_{N}\right)^{-1}}{w^{n}\left(\lambda_{k, N}-w\right)\left(\lambda_{\ell, N}-w\right)} \mathrm{d} w,
$$

with $\mathcal{C}$ any circle centered at 0 with radius in the interval $\left(\sigma^{2} c_{N}, \sigma^{2} \sqrt{c_{N}}\right)$. Define also

$$
\Gamma_{N}(k, \ell)=\operatorname{Re}\left(\eta_{k, N}^{(1,2)} \eta_{\ell, N}^{(1,2)}\right)+\frac{\eta_{k, N}^{(1,1)} \eta_{\ell, N}^{(2,2)}+\eta_{\ell, N}^{(1,1)} \eta_{k, N}^{(2,2)}}{2}
$$

${ }^{1}$ An explicit expression can be obtained for $\vartheta_{N}(k, \ell)$, by solving the integral $I_{n, N}(k, \ell)$ with residue theorem, but this representation is kept due to lack of space. 
where $\eta_{k, N}^{(i, j)}=\mathbf{d}_{i, N}^{*} \mathbf{u}_{k, N} \mathbf{u}_{k, N}^{*} \mathbf{d}_{j, N}$. Finally, we set

$$
\Gamma_{N}=\sum_{k=1}^{M} \sum_{\ell=1}^{M} \vartheta_{N}(k, \ell) \Gamma_{N}(k, \ell) .
$$

Theorem 2. If $\liminf \operatorname{in}_{N \rightarrow \infty} \Gamma_{N}>0$, then

$$
\frac{\sqrt{N} \operatorname{Re}\left(\hat{\eta}_{N}-\eta_{N}\right)}{\sqrt{\Gamma_{N}}} \underset{N \rightarrow \infty}{\stackrel{\mathcal{D}}{\longrightarrow}} \mathcal{N}_{\mathbb{R}}(0,1) .
$$

\section{Performance ANAlysis of BeAmSPaCe-MUSIC}

We recall that the general idea of the Beamspace-MUSIC method consists in applying the classical MUSIC method on the "beamformed" observations matrix

$$
\tilde{\mathbf{Y}}_{N}=\mathbf{B}_{N}^{*} \mathbf{Y}_{N}
$$

where $\mathbf{B}_{N}$ is a $M \times L$ matrix with orthonormal columns. Hence, the classical "element-space" model (1) reduces to the "beamspace" model with lower dimension $L$

$$
\tilde{\mathbf{Y}}_{N}=\tilde{\mathbf{A}} \mathbf{S}_{N}+\tilde{\mathbf{V}}_{N}
$$

where $\tilde{\mathbf{V}}_{N}=\left[\tilde{\mathbf{v}}_{1}, \ldots, \tilde{\mathbf{v}}_{N}\right]$ with $\tilde{\mathbf{v}}_{1}, \ldots, \tilde{\mathbf{v}}_{N}$ i.i.d. $\mathcal{N}_{\mathbb{C}^{L}}\left(\mathbf{0}, \sigma^{2} \mathbf{I}\right)$, and where $\tilde{\mathbf{A}}=\left[\tilde{\mathbf{a}}\left(\theta_{1}\right), \ldots, \tilde{\mathbf{a}}\left(\theta_{K}\right)\right]$ is the beamformed steering vectors matrix, with $\tilde{\mathbf{a}}(\theta)=\mathbf{B}_{N}^{*} \mathbf{a}(\theta)$. The beamspace MUSIC algorithm then consists in estimating the DoA $\theta_{1}, \ldots, \theta_{K}$ as the $K$ most significant minima of

$$
\hat{\eta}_{N}^{(b)}(\theta)=\tilde{\mathbf{a}}(\theta)^{*} \hat{\boldsymbol{\Pi}}_{N}^{(b)} \tilde{\mathbf{a}}(\theta)
$$

where $\hat{\boldsymbol{\Pi}}_{N}^{(b)}$ is the orthogonal projection matrix onto the eigenspace associated with the $L-K$ smallest eigenvalues of the $\operatorname{SCM~} N^{-1} \tilde{\mathbf{Y}}_{N} \tilde{\mathbf{Y}}_{N}^{*}$.

We consider here the DFT beamformer, obtained by selecting a set of columns from a DFT matrix. Usually, it is assumed that the sources DoA are located in some spatial sector $\Theta$, that is, a compact subset of $[-\pi, \pi]$, which is assumed known. In that case, by defining

$$
\mathcal{V}_{N}=\left\{-\pi+\frac{2 \pi m}{M}: m=0, \ldots, M-1\right\},
$$

and $\left\{\nu_{1, N}, \ldots, \nu_{L, N}\right\}=\Theta \cap \mathcal{V}_{N}$, we consider the beamforming matrix

$$
\mathbf{B}_{N}=\left[\mathbf{a}\left(\nu_{1, N}\right), \ldots, \mathbf{a}\left(\nu_{L, N}\right)\right] .
$$

Of course, such a choice of $\mathbf{B}_{N}$ ensures that $\tilde{\mathbf{A}}=\mathbf{B}_{N}^{*} \mathbf{A}$ is full-rank $K$ as long as $L \geq K$.

For the remainder, we assume ${ }^{2}$ that the DoA $\theta_{1}, \ldots, \theta_{K}$ are independent of $N$. Moreover, we assume that the angular sector $\Theta$ is such that $\theta_{1}, \ldots, \theta_{K} \in \operatorname{Int}(\Theta)$, where $\operatorname{Int}(\Theta)$ is the interior of $\Theta$, and that $\Theta$ is the union of a fixed number of compact intervals, with Lebesgue measure $\Delta \in(0,2 \pi]$. In that case, we have

$$
d_{N}=\frac{L}{N} \underset{N \rightarrow \infty}{\longrightarrow} d=\frac{\Delta}{2 \pi} c .
$$

\footnotetext{
${ }^{2}$ In practice, for finite $N$, this would model a situation where the source DoA are widely spaced.
}

To study the consistency and asymptotic Gaussianity of the Beamspace-MUSIC DoA estimates, we essentially rely on the results of section II, by setting this time $\boldsymbol{\Sigma}_{N}=N^{-1 / 2} \tilde{\mathbf{Y}}_{N}$, $\mathbf{X}_{N}=N^{-1 / 2} \tilde{\mathbf{A}} \mathbf{S}_{N}$ and $\mathbf{W}_{N}=N^{-1 / 2} \tilde{\mathbf{V}}_{N}$, and replacing $M, c_{N}, c$ by $L, d_{N}, d$.

Remark 1. Of course, if $\Theta=[-\pi, \pi]$, then $L=M$ and the Beamspace-MUSIC coincides exactly with MUSIC, that is $\hat{\eta}_{N}^{(b)}(\theta)=\hat{\eta}_{N}(\theta)$ for all $\theta \in[-\pi, \pi]$. Therefore, the subsequent analysis will also encompass the traditional MUSIC.

The following property on the asymptotic orthonormality of the beamformed steering vectors will be useful.

Lemma 1. Let $\mathcal{I} \subset \operatorname{Int}(\Theta)$ be a closed interval. Then, $\sup _{\theta \in \mathcal{I}}\left|\|\tilde{\mathbf{a}}(\theta)\|^{2}-1\right|=\mathcal{O}\left(N^{-1}\right)$ and for all $\psi \in \operatorname{Int}(\Theta) \backslash \mathcal{I}$,

$$
\sup _{\theta \in \mathcal{I}}\left|\tilde{\mathbf{a}}(\theta)^{*} \tilde{\mathbf{a}}(\psi)\right|=\mathcal{O}\left(\frac{1}{N}\right) .
$$

Moreover, $\operatorname{Re}\left(N^{-1} \tilde{\mathbf{a}}^{\prime}\left(\theta_{k}\right) \mathbf{u}_{\ell, N} \mathbf{u}_{\ell, N}^{*} \tilde{\mathbf{a}}\left(\theta_{k}\right)\right)=\mathcal{O}\left(N^{-1}\right)$ for $k, \ell \in\{1, \ldots, K\}$.

We also make the following assumption.

Assumption 2. The following convergence holds

$$
\frac{\mathbf{S}_{N} \mathbf{S}_{N}^{*}}{N} \underset{N \rightarrow \infty}{\longrightarrow} \boldsymbol{\Lambda},
$$

where $\boldsymbol{\Lambda}=\operatorname{diag}\left(\lambda_{1}, \ldots, \lambda_{K}\right)$ where $\lambda_{1}>\ldots>\lambda_{K}>\sigma^{2} \sqrt{d}$.

Under Assumption 2, and using Lemma 1, we notice that $\tilde{\mathbf{A}}^{*} \tilde{\mathbf{A}}=\mathbf{I}+\mathcal{O}\left(N^{-1}\right)$ and the $K$ non-zero eigenvalues $\lambda_{1, N}, \ldots, \lambda_{K, N}$ of $N^{-1} \tilde{\mathbf{A}} \mathbf{S}_{N} \mathbf{S}_{N}^{*} \tilde{\mathbf{A}}^{*}$ converge to $\lambda_{1}, \ldots, \lambda_{K}$. Therefore, the separation condition (Assumption 1) is satisfied in this situation.

Remark 2. At this point, we remark that the SNR required for the subspace separation condition to hold has lowered to $\sqrt{\frac{\Delta}{2 \pi} c}$ instead of $\sqrt{c}$, if we define the SNR as the ratio $\frac{\lambda_{K}}{\sigma^{2}}$.

In the remainder, we use the notation $\left(\epsilon_{N}\right)$ for a generic deterministic error term vanishing at rate $\mathcal{O}\left(\left\|\frac{\mathbf{s}_{N} \mathbf{S}_{N}^{*}}{N}-\boldsymbol{\Lambda}\right\|\right)+$ $\mathcal{O}\left(N^{-1}\right)$ (the value of $\epsilon_{N}$ can change from one line to another). Under Assumption 2, we retrieve the usual asymptotic orthonormality between steering vectors and eigenvectors. Indeed, $\left|\mathbf{a}\left(\theta_{k}\right)^{*} \mathbf{u}_{\ell, N}\right|=\delta_{k, \ell}+\epsilon_{N}$, while for any fixed interval $\mathcal{I} \subset \operatorname{Int}(\Theta)$ such that $\theta_{k} \notin \mathcal{I}, \sup _{\theta \in \mathcal{I}}\left|\tilde{\mathbf{a}}(\theta)^{*} \mathbf{u}_{k, N}\right|=\epsilon_{N}$. Moreover, the following approximations also hold.

Lemma 2. Under Assumption 2,

$$
\begin{aligned}
& \frac{\tilde{\mathbf{a}}^{\prime}\left(\theta_{k}\right)^{*}}{N} \mathbf{u}_{\ell, N} \mathbf{u}_{\ell, N}^{*} \tilde{\mathbf{a}}\left(\theta_{k}\right)=-\mathrm{i} \frac{c}{2} \delta_{k, \ell}+\epsilon_{N}, \\
& \left|\frac{\tilde{\mathbf{a}}^{\prime}\left(\theta_{k}\right)^{*}}{N} \mathbf{u}_{\ell, N}\right|=\frac{c}{2} \delta_{k, \ell}+\epsilon_{N}, \\
& \frac{\tilde{\mathbf{a}}^{(2)}\left(\theta_{k}\right)}{N^{2}} \mathbf{u}_{\ell, N} \mathbf{u}_{\ell, N}^{*} \mathbf{a}\left(\theta_{k}\right)=-\frac{c^{2}}{3} \delta_{k, \ell}+\epsilon_{N} .
\end{aligned}
$$

Let $\mathcal{I}_{1}, \ldots, \mathcal{I}_{K}$ be compact disjoint intervals subset of $\operatorname{Int}(\Theta)$ and containing respectively $\theta_{1}, \ldots, \theta_{K}$. We define 
formally the Beamspace-MUSIC DoA estimates as

$$
\hat{\theta}_{k, N}^{(b)}=\underset{\theta \in \mathcal{I}_{k}}{\operatorname{argmin}} \hat{\eta}_{N}^{(b)}(\theta),
$$

for $k=1, \ldots, K$. Using Theorem 1 with $\mathbf{d}_{1, N}=\mathbf{d}_{2, N}=\tilde{\mathbf{a}}(\theta)$ and applying the procedure used in [6, Th. 3], we obtain the following uniform convergence

$$
\sup _{\theta \in \Theta}\left|\hat{\eta}_{N}^{(b)}(\theta)-\eta_{N}^{(b)}(\theta)\right| \underset{N \rightarrow \infty}{\stackrel{a . s .}{\longrightarrow}} 0
$$

where $\eta_{N}^{(b)}(\theta)=\|\tilde{\mathbf{a}}(\theta)\|^{2}-\sum_{k=1}^{K} \gamma_{k, N}\left|\tilde{\mathbf{a}}(\theta)^{*} \mathbf{u}_{k, N}\right|^{2}$. Moreover, using Lemma 2, we further obtain

$$
\sup _{\theta \in \mathcal{I}_{k}}\left|\eta_{N}^{(b)}(\theta)-\left(1-\gamma_{k, N}\left|\tilde{\mathbf{a}}(\theta)^{*} \tilde{\mathbf{a}}\left(\theta_{k}\right)\right|^{2}\right)\right| \underset{N \rightarrow \infty}{\stackrel{a . s .}{\longrightarrow}} 0,
$$

while $\sup _{\theta \in \Theta \backslash \bigcup_{k} \mathcal{I}_{k}} \eta_{N}^{(b)}(\theta) \rightarrow 1$. Since function $\theta \mapsto$ $\left|\tilde{\mathbf{a}}(\theta)^{*} \tilde{\mathbf{a}}\left(\theta_{k}\right)\right|^{2}$ has a unique global maximum at $\theta_{k}$, we deduce that $\hat{\theta}_{k, N}^{(b)} \rightarrow \theta_{k}$ a.s. Finally, using a similar analysis as in [6, Th. 3], we can prove the following refinement.

Theorem 3. Under Assumption 2, for all $k=1, \ldots, K$,

$$
\hat{\theta}_{k, N}^{(b)}=\theta_{k}+o\left(\frac{1}{N}\right) \quad \text { a.s. }
$$

Concerning the 2 nd order analysis, we follow the standard steps of M-estimation, based on a Taylor expansion of $\hat{\eta}_{N}^{(b)^{\prime}}(\theta)$ around $\theta_{k}$ (see e.g. [6]). Using Theorem 1 and 3, we obtain

$$
N^{3 / 2}\left(\hat{\theta}_{k, N}^{(b)}-\theta_{k}\right)=-\frac{N^{-1 / 2} \hat{\eta}_{N}^{(b)^{\prime}}\left(\theta_{k}\right)}{N^{-2} \eta_{N}^{(b)(2)}\left(\theta_{k}\right)+o(1)} \quad \text { a.s. }
$$

Finally, Lemma 1 gives $N^{-1} \eta_{N}^{(b)^{\prime}}\left(\theta_{k}\right)=\mathcal{O}\left(N^{-1}\right)$, and using Theorem 2 with $\mathbf{d}_{1, N}=N^{-1} \tilde{\mathbf{a}}^{\prime}\left(\theta_{k}\right)$ and $\mathbf{d}_{2, N}=\tilde{\mathbf{a}}\left(\theta_{k}\right)$, we deduce

$$
\frac{\hat{\eta}_{N}^{(b)^{\prime}}\left(\theta_{k}\right)}{2 \sqrt{N} \sqrt{\Gamma_{N}}} \underset{N \rightarrow \infty}{\stackrel{\mathcal{D}}{\longrightarrow}} \mathcal{N}_{\mathbb{R}}(0,1)
$$

Therefore, the following result holds.

Theorem 4. Under Assumption 2, for any $k=1, \ldots, K$,

$$
N^{3 / 2} \frac{N^{-2} \eta_{N}^{(b)(2)}\left(\theta_{k}\right)}{2 \sqrt{\Gamma_{N}}}\left(\hat{\theta}_{k, N}^{(b)}-\theta_{k}\right) \underset{N \rightarrow \infty}{\stackrel{\mathcal{D}}{\longrightarrow}} \mathcal{N}_{\mathbb{R}}(0,1) .
$$

Using the approximations of Lemma 2, we can obtain a more compact expression for the MSE term in (12). Indeed,

$$
\eta_{N}^{(2)(b)}\left(\theta_{k}\right)=\frac{c^{2}}{6} \frac{\lambda_{k}^{2}-\sigma^{4} d}{\lambda_{k}\left(\lambda_{k}+\sigma^{2} d\right)}+\epsilon_{N} .
$$

From Lemma 2, we remark that $\Gamma_{N}(k, \ell)=o(1)$ for $k, \ell \in$ $\{1, \ldots, K\}$, and consequently, we can prove that

$$
\Gamma_{N}=\frac{c^{2}}{24} \frac{\sigma^{2}\left(\lambda_{k}+\sigma^{2}\right)\left(\lambda_{k}^{2}-\sigma^{4} d\right)}{\lambda_{k}^{2}\left(\lambda_{k}+\sigma^{2} d\right)^{2}}+\epsilon_{N} .
$$

Finally, Theorem 4 rewrites

$$
N^{3 / 2} \sqrt{\frac{c^{2}}{6} \frac{\left(\lambda_{k}^{2}-\sigma^{4} d\right)}{\sigma^{2}\left(\lambda_{k}+\sigma^{2}\right)}}\left(\hat{\theta}_{k, N}^{(b)}-\theta_{k}\right) \underset{N \rightarrow \infty}{\stackrel{\mathcal{D}}{\longrightarrow}} \mathcal{N}_{\mathbb{R}}(0,1) .
$$

Remark 3. From the convergence (14), we notice that the knowledge of an angular sector $\Theta$, symbolized by its length $\Delta \leq 2 \pi$, gives a reduced asymptotic MSE, compared to the one we would have without beamforming (traditional MUSIC), since $d \leq c$.

In Figure 1, we plot the empirical MSE of BeamspaceMUSIC and G-MUSIC [2] against the SNR, defined as $-10 \log \left(\sigma^{2}\right)$, for 2 sources. The entries of the signal matrix $\mathbf{S}_{N}$ are the realization of independent standard complex Gaussian variables. The theoretical MSE predicted by Theorem 4 is also represented, as well as the Cramer-Rao Bound (CRB). The SNR required for the subspace separation condition to hold is visible through the so-called threshold effect, which occurs for a lower SNR in the case of Beamspace-MUSIC (around $-4 \mathrm{~dB}$ against $2 \mathrm{~dB}$ for G-MUSIC).

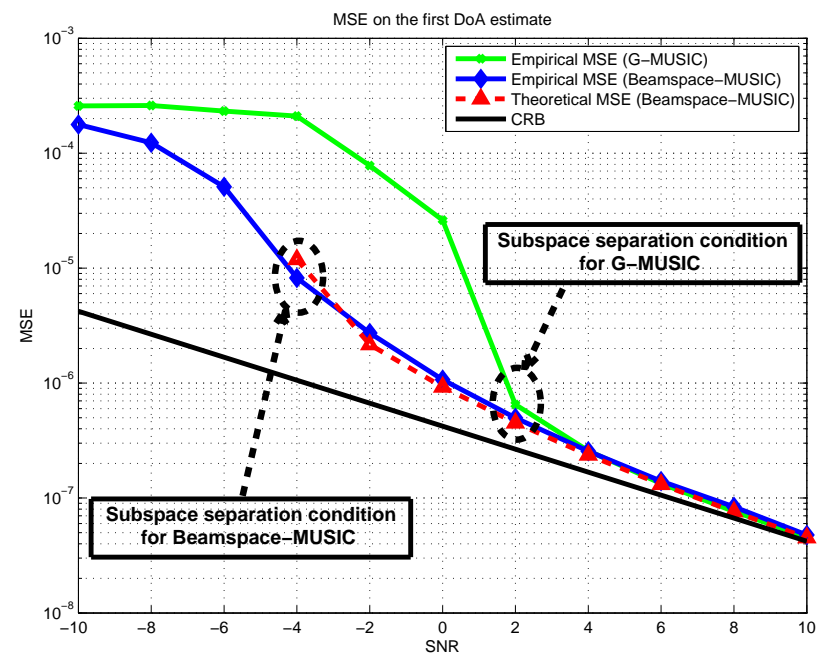

Figure 1. $M=200, N=400, c_{N}=0.5, \theta_{1}=0, \theta_{2}=0.05, d_{N} \approx 0.08$

\section{REFERENCES}

[1] X. Mestre, "Improved estimation of eigenvalues and eigenvectors of covariance matrices using their sample estimates," IEEE Trans. Inf. Theory, vol. 54, no. 11, pp. 5113-5129, 2008.

[2] P. Vallet, P. Loubaton, and X. Mestre, "Improved subspace estimation for multivariate observations of high dimension: The deterministic signal case," IEEE Trans. Inf. Theory, vol. 58, no. 2, Feb. 2012.

[3] A. J. Weiss and B. Friedlander, "Preprocessing for direction finding with minimal variance degradation," IEEE Trans. Signal Process., vol. 42, no. 6, pp. 1478-1485, 1994.

[4] A. Thakre, M. Haardt, and K. Giridhar, "Single snapshot spatial smoothing with improved effective array aperture," IEEE Signal Processing Letters, vol. 16, no. 6, pp. 505-508, 2009.

[5] Y. Wang, G. Leus, and A. Pandharipande, "Direction estimation using compressive sampling array processing," in Proceedings of 2009 IEEE Statistical Signal Processing Workshop (SSP), 2009, pp. 626-629.

[6] W. Hachem, P. Loubaton, X. Mestre, J. Najim, and P. Vallet, "A subspace estimator for fixed rank perturbations of large random matrices," $J$. Multivariate Anal., vol. 114, pp. 427-447, 2013.

[7] P. Vallet, X. Mestre, and P. Loubaton, "A clt for the g-music doa estimator," in Proceedings of the 20th European Signal Processing Conference (EUSIPCO). IEEE, 2012, pp. 2298-2302. 